\title{
Design and Implementation of Remote Control Software for Nuclear Inspecting Devices
}

\author{
Chengze Liu, Yi Zhang, Baoliang Cheng, Xiaomin Zhao, Ran Duan \\ State Nuclear Power Plant Service Company \\ Shanghai, China \\ e-mail: chengzeliu@gmail.com
}

\begin{abstract}
Remote control systems are usually adopted in nuclear facilities inspecting activities due to radioactive environments. Analysis on requirements and structural design are made, and functional modules are described in detail. Remote control software for nuclear equipment inspection is developed with combination of OpenGL 3-dimensional graphics and virtual reality technologies. Operators may try offline simulation before sending commands to control hardware, which improves accuracy and reliability of remote control system.
\end{abstract}

Keywords-nuclear power plant; remote control; virtual reality; 3-dimensional display

\section{INTRODUCTION}

According to corresponding guideline, periodic inspection should be executed for nuclear important facilities such as reactor pressure vessel, steam generator and so on, which is to ensure structural integrity. Those facilities like reactor pressure vessel usually work in highly radioactive environment, so remote maneuver control system is necessary to implement high-precision automatic inspection. The surrounding environment is complicated, and limited time window is restricted to complete preset task movement, which put forward demand for high reliability and accuracy. It's the key to remote maneuver control system that operators can justify following operation accurately and reliably. To avoid operation mistake, simulation for task execution is necessary before executing actual movement. Software simulation is helpful for ensuring functional validation and space accessibility.

In recent years researchers have paid much attention to remote maneuver control software technologies for nuclear facilities inspection. Antal K.Bejczy reveals that computer graphics can be utilized in task visualization, motion preview, actual operation anticipation, staff training and etc ${ }^{[1]}$. Seungho Kim and his colleagues research the application of robotics on nuclear power plant maintenance. 3-dimensional graphics is utilized to develop the interface between monitoring PC and controller ${ }^{[2]}$, and 3-dimensional ADAM mechanical models for robot arm and site components are developed, which can be used in conceptual design, detailed path planning and operation training. Laurent Chodorge and his staffs developed a set of simulation tool software CHAVIR. CHAVIR may help operators to simulate the manual operations, and estimate the dose rate after importing
AutoCAD models. In addition, CHAVIR can also simulate physical situation, confirm whether it is accessible or not ${ }^{[3]}$. In addition, Patricia Garcia and his staffs have focused their research on remote control platform needed by nuclear fueling facilities maintenance ${ }^{[4]}$.

Remote maneuver control software for nuclear facilities inspecting device is analyzed in the paper. Software systematic structure is brought up. OpenGL 3D graphic technology and VRML virtual reality are combined to implement the remote maneuver software.

\section{SYSTEMATIC DESCRIPTION}

Due to highly radioactive environments in nuclear power plant, research is mainly focused on a typical remote control system for nuclear facility-inspecting devices. The hardware mainly consists of onsite control hardware, actuators, instruments and remote operating computer, as can be seen in Fig.1. Data from controller output is directly delivered to remote actuators, but some unsteady elements may exist in key factors, such as time delay, occasional collision and etc. So, if motion plan can be modeled and confirmed before sending to actuators, influence of unsteady elements can be diminished to some extent at least. Once the operator gets enough modeling information, he can send the control command to simulating functional block to validate. After validating, operators may send command to remote actuators to ensure safe movement.

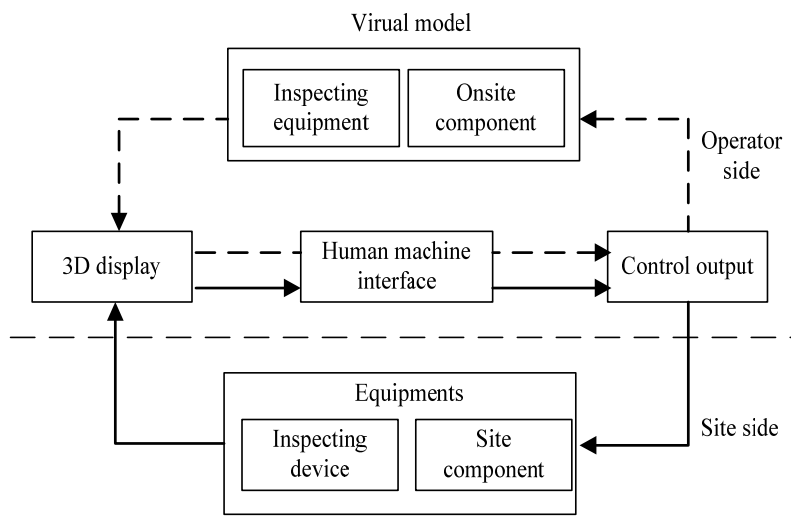

Figure 1. Sketch of Remote control System

The control system is mainly composed of remote controlling computer, onsite controller, sensors, transducers and etc. The key to computer control system is software 
designing and implementation. The following function parts should be included in software.

- Motion control. The inspecting device should be controlled to move along preset path and complete task execution.

- Site simulation. 3-dimensional simulation display interface should be provided to confirm the accessibility of working space.

- Operation simulation. Simulation for software verification and validation is needed to ensure safe movement.

- Interaction with operators. HMI should be simple but definite for easy operation.

\section{SOFTWARE STRUCTURE}

According to software engineering, remote maneuver control software can be divided into several functional modules including hardware interface, simulating computation, data pretreated interface, path calculation, path tracking, 3D display, model resolving, and status monitoring. Each functional module is independent which is convenient for software maintenance and expansion. Overall sketch is shown in Fig.2.

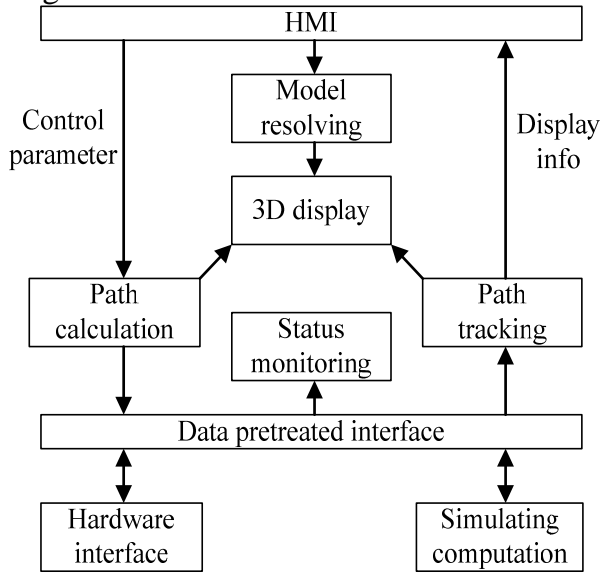

Figure 2. Sketch of Software Structure

Functional modules are described in detail as follows.

1) Hardware interface module. Control commands with preset form are sent to controller hardware to drive actuators follow task instructions. Data from sensors on inspecting device are sent back to hardware interface at same time for monitoring site situation. These data will be delivered to data pretreated interface.

2) Simulating computation. This module is used for operation validation before task execution in case of operation mistake or collision with site components. Functions involved may include calculating virtual position, velocity according to input parameters, which will be delivered to data pretreated interface.

3) Data pretreated interface. This module belongs to middleware program. Main function is to distribute feedback data from hardware interface or simulating computation module and sends them to path tracking, monitoring or others. At same time, result data from path computation module will be accepted, reassembled, then sent to hardware interface or simulating computation in unified form.

4) Path computation. Path points are calculated according to customized task parameters, input command and other component parameters, which can be translated to axes' coordinate values. And these values will be sent to data interface module for executing.

5) 3D dynamic display. Data from different sources are accepted, depending on operating mode. OpenGL display list is utilized to render 3-dimensional image. The module accepts data from model resolving, and generates the display list. When OpenGL display list is finished, the screen display will be refreshed as released. Display list may be dynamically rebuilt when path info is modified or new component model is selected, then the display will also be refreshed.

6) Model resolving. Model involved includes inspecting device, inspecting tools and site components. It will be complicated and unnecessary for modeling with OpenGL, so model resolving is utilized for importing model files from Inventor or Solidworks software. VRML has been standard format describing virtual scene with development of virtual reality ${ }^{[5]}$. VRML model file is selected as universal format in the software. Data structure is designed according to object data in VRML files. Several classes are built to restore data from VRML files. For instance, CVRPointSet class is built to store point data including point coordinates, color and etc. CVRLineSet class is built to store line data including index, color and other features. While CVRFaceSet is built to store face data, including face index, normal vector and so on. General data such as geometry, appearance and transformation data are resolved and restored in preset variables.

7) Status monitoring. It accounts for status monitoring, and status data includes current axes position, load torque, movement error, and system heart beating status. All data will be feed back to operators for screen display or alarm hint. In addition, monitoring module also accounts for detecting abnormal operation, run away and emergency situation. Once it happens, corresponding functional subroutine will be called to recover from malfunction or stop midway.

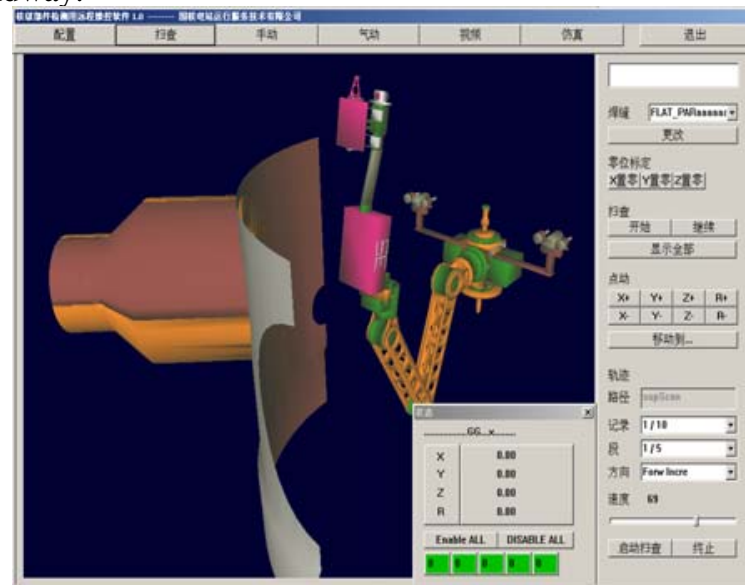

Figure 3. Operating Interface 
Remote maneuver control software for nuclear facility inspection is implemented based on modules mentioned above. It runs good so far, operating interface as shown in Fig.3.

\section{ANALYSIS ON KEY TECHNOLOGIES}

Key technologies involved include VRML file resolving, OpenGL 3-dimensional display and reliability design.

\section{A. VRML file resolving}

Complex geometries in VRML file are described with indexed face set node. Each face set node has the same composing structure, corresponding to one entity object. Once node is validated, the face set object will be created, and the storage process will start. Entity data will be refreshed in node list. According to nesting feature of layers, recursive data fetching is adopted from beginning entity to last one. Once the VRML file is finished, the display list will be released.

\section{B. OpenGL 3-dimensional display}

OpenGL display list mode is adopted to accelerate image rendering. Display list is the high-speed cache memory. Once the display list is triggered, the list will be executed with released sequence. Creation of display list is shown in Fig.4. The list will be dynamically rebuilt when path info is modified and new component model is selected or supplemented.

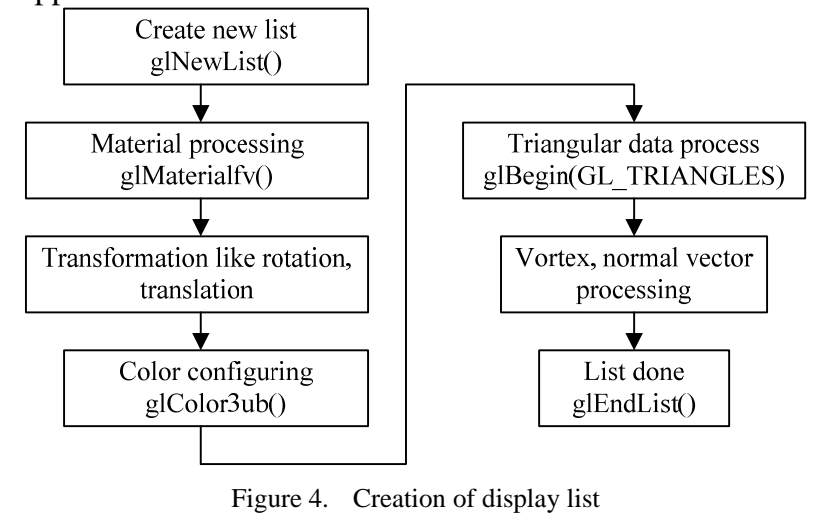

In addition, properties including environment lightness, diffusing lightness and mirror reflecting are defined to improve realistic display.

\section{Reliability analysis}

As for the nuclear facility inspection, the inspecting device usually works in radioactive environment which is dangerous for workers to access. If the remote system didn't work, it will be much difficult for maintenance and repairing. So, highly reliability has to be ensured. As far as software designing is concerned, some measurements as following are adopted to improve reliability.

- Exception handling. Exception capturing and handling codes are added in important source codes, which is to avoid the software run out of control;
- Classification for real time demand. All function modules are divided into hard real time, soft real time and non-realtime. The hardware interface, status monitoring module are hard real time tasks, the controlling recursive period set to $20 \mathrm{~ms}$. Path computation is soft real-time task, control period set to $100 \mathrm{~ms}$. 3-dimensional display is non-realtime task, controlling period set to $200 \mathrm{~ms}$.

- Path tracking. Task executing process should be tracked during running time. Once deviation from preset path is captured, the software will try to correct the deviation to keep the error into admitted range. If correction cannot be done, path tracking module will notify the main program with alarm hint and stop movement.

\section{CONCLUSION}

Remote maneuver control has to be adopted in nuclear site facilities inspection due to radioactive environment, which demands for highly reliability and performance.

Research on requirements analysis and structural design is made for nuclear inspecting remote control software, and functional modules are analyzed. OpenGL 3D graphics and VRML virtual reality are combined to realize the software. With switching between simulation and actual movement mode, operators may simulate task execution before sending command to actual actuator, which enhance accuracy and reliability of remote maneuver control. In addition, the remote maneuver software may apply to many general applications with related customization, such as nuclear power plant in-service inspection or decontamination cleaning.

\section{ACKNOWLEDGMENT}

The authors wish to thank State Nuclear Power Plant Service Company for providing experiment conditions and other colleagues for providing data and drawings.

\section{REFERENCES}

[1] Antal K.Bejczy, Won Soo Kim, Paul S.Schenker. "The Role of Computer Graphics in TeleoperationRobert Bogue. Robots in the Nuclear Industry: a Review of Technologies". Robotics for Challenging environments, 1994, New Mexico, America, pp. 1-9

[2] Seungho Kim, Seung Ho Jung, Chang Hoi Kim. "Preventive Maintenance and Remote Inspection of Nuclear Power Plants Using Tele-robotics". Proceedings of the 1999 IEEE/RSJ International Conference on Intelligent Robots and Systems, 1999, Korea, pp. 603608

[3] Laurent Chodorge, Arnauld Leservot, Philippe Desbats. "CHAVIR a virtual reality simulation environment for hazardous nuclear working sites”. 1st joint emergency preparedness and response / robotic and remote systems, 2006, USA, pp. 516-523

[4] Patricia Garcia-Borras, Pablo Garcia Robledo, Jorge Barrio. "Technofusion Remote Handling Laboratory: Contributions to Nuclear Fusion Facilities Maintenance Tasks”. 2010 1st International Conference on Applied Robotics for the Power Industry, 2010, Canada, pp. 1-6

[5] Zhang Zhengxia, Wang Houxiang. Outline and Prospect of VRML Journal of Engineering Graphic, 1998, Vol.2, pp. 85-91 